\title{
Faire réussir les filles. Prescriptions et réalités
}

Getting girls to succeed: Prescriptions and realities

Promover el éxito de las chicas en la escuela. Prescripciones y realidades

\section{Patrick Rayou}

\section{OpenEdition}

\section{Journals}

Édition électronique

URL : https://journals.openedition.org/ries/9311

DOI : $10.4000 /$ ries. 9311

ISSN : 2261-4265

\section{Éditeur}

France Education international

Édition imprimée

Date de publication : 30 avril 2020

Pagination : 69-73

ISBN : 978-2-85420-626-5

ISSN : $1254-4590$

\section{Référence électronique}

Patrick Rayou, «Faire réussir les filles. Prescriptions et réalités 》. Revue internationale d'éducation de Sèvres [En ligne], 83 | avril 2020, mis en ligne le 17 juin 2020, consulté le 25 juin 2021. URL : http:// journals.openedition.org/ries/9311; DOI : https://doi.org/10.4000/ries.9311 


\title{
Faire réussir les filles. Prescriptions et réalités
}

\author{
Patrick Rayou \\ Université Paris 8, Circeft-Escol
}

Toutes les études internationales montrent les bénéfices que tireraient non seulement les femmes, mais l'humanité entière d'une meilleure scolarisation des filles. Quelques exemples pris parmi la nombreuse littérature disponible :

Si chaque fille dans le monde bénéficiait de 12 années de scolarisation de qualité, les revenus des femmes pourraient augmenter de 15 à 30 trillions de dollars à l'échelle mondiale. (Source : Missed opportunities: the high cost of not educating girls)

La richesse du capital humain pourrait augmenter de près de $22 \%$ à l'échelle mondiale, si les revenus des femmes étaient égaux à ceux des hommes. (Source : Unrealized potential: the high cost of gender inequality in earnings)

Éduquer des filles a permis d'éviter plus de 30 millions de décès d'enfants de moins de cinq ans et 100 millions de décès d'adultes de 15 à 60 ans. (Source : The Learning Generation)

Pourtant, selon l'Unesco', ce sont près de 16 millions de filles âgées de 6 à 11 ans qui ne fréquenteront jamais l'école primaire, contre environ 8 millions de garçons, si la tendance actuelle se poursuit. Irina Bokova, quand elle était sa directrice, a déclaré :

Nous n'atteindrons jamais les Objectifs de développement durable, si nous ne luttons pas contre la discrimination et la pauvreté qui affectent la vie des filles et des femmes d'une génération à l'autre (...). Nous devons travailler à tous les niveaux, des populations jusqu'aux dirigeants, pour inscrire l'égalité et l'inclusion au cœur de chaque politique, afin que toutes les filles, quelle que soit leur situation, aillent à l'école, restent à l'école et deviennent des citoyennes autonomes².

Le volontarisme en la matière s'impose si l'humanité souhaite vraiment assurer l'égalité des genres, voire, dans un avenir plus proche que prévu, assurer sa propre survie. Pourtant, même dans des pays où le législateur s'efforce de prendre et de faire appliquer des mesures en ce sens, les résultats sont souvent mitigés, voire décevants. Une étude de deux cas, ceux du Mali et de la France ${ }^{3}$, peut donner des éléments de compréhension de ces phénomènes qui, bien que très dépendants de contextes différents, éclairent sur des difficultés structurelles à faire réussir des réformes dont l'utilité est pourtant incontestable.

1. Atlas de l'Unesco. [https://bit.ly/2u2tjLo]

2. Déclaration du 2 mars 2016.

3. Ces études de cas ont été présentées lors de l'atelier « Mettre en œuvre les réformes, acceptabilité et mobilisations » tenu lors du colloque "Conditions de réussite des réformes en éducation » organisé par la Revue internationale d'éducation de Sèvres, 12-14 juin 2019, CIEP. [https://journals.openedition.org/ries/6658] 


\section{Des aVANCÉES DANS LA MIXITÉ}

Au Mali, depuis la proclamation de l'indépendance en 1960, l'objectif de scolarisation est porté par de nombreuses réformes. Celle de 1962, notamment, visant un enseignement de masse et de qualité pour tous, ou, plus près de nous, en 1998, le Programme décennal de développement de l'éducation (PRODEC), qui fixe comme objectif d'atteindre, à l'horizon 2010, un taux brut de scolarisation de $95 \%$ au premier cycle de l'enseignement fondamental. Adoptées par les autorités politiques et scolaires, ces réformes, qui visaient notamment à améliorer la situation éducative des filles, ont eu le soutien de partenaires comme la Banque mondiale, l'Unesco, l'ONG Save the children ou le Programme alimentaire mondial. Elles ont en partie atteint leur but, si l'on prend pour critère les résultats obtenus lors de la validation du diplôme d'études fondamentales (DEF) qui sanctionne neuf ans d'études primaires, à l'issue desquelles les élèves peuvent aller vers un second cycle d'enseignement général ou technique et professionnel. La disparité entre filles et garçons s'est en effet progressivement atténuée : avec un taux de réussite de 44,3\% en 2015 (pour 51,3\% de garçons), de 63,4 \% en 2015 (pour 74,8\%), elles atteignent presque la parité en 2018 , avec $60,4 \%$ (pour $61,4 \%$ ). Au baccalauréat général, les taux étaient respectivement de $28,34 \%$ et $28,71 \%{ }^{5}$.

En France, longtemps réservé aux élites masculines, le baccalauréat, certificat de fin d'études secondaires et premier grade universitaire, s'est ouvert aux jeunes filles au début des années 1920. Cette volonté de mixité a néanmoins pris plusieurs décennies pour se concrétiser. Au sortir de la Deuxième Guerre mondiale, les idéaux d'égalité qui ont alors façonné l'école se sont trouvés en phase avec l'entrée, progressive mais massive, des femmes dans la production. Ce à tel point que, très minoritaires au début du $\mathrm{xx}^{\mathrm{e}}$ siècle dans le secondaire et le supérieur, elles ont égalé en nombre les étudiants à la fin des années 1970 pour les dépasser ensuite, qu'il s'agisse de la durée moyenne des études, du niveau moyen de diplômes, du taux de réussite aux examens, du niveau moyen aux épreuves de contrôle, des acquis scolaires, etc. En 1999, les femmes sont devenues majoritaires en troisième cycle universitaire. Le pourcentage des femmes ingénieurs a pratiquement doublé entre 1984 et 2008, passant de $15 \%$ à $26 \%$, tandis que les diplômées des écoles de commerce sont passées de $38 \%$ à $48 \%$. Les deux tiers de la croissance des effectifs étudiants depuis 1990 sont dus aux femmes. Alors que la proportion des femmes nées au cours des années 1950 et détentrices d'un bac +2 est inférieure à celle des hommes, la génération des femmes de 25-34 ans représente $25 \%$ des diplômés de l'enseignement supérieur contre $20 \%$ des hommes du même âge 6 .

\section{DES SUCCÈs RELATIFS}

Ces données générales peuvent néanmoins recouvrir de grandes disparités. Ainsi, le taux d'obtention du DEF des filles maliennes est de 81,1\% en milieu urbain pour $54,7 \%$ en milieu rural, où vivent les trois quarts de la population.

\footnotetext{
4. Institut national de statistique, 2017 ; Ministère de l'éducation nationale, 2018.

5. Centre national des examens et concours de l'éducation, 2018.

6. Conseil économique, social et environnemental, 2009.
} 
À la campagne, beaucoup de femmes demeurent non lettrées, subordonnées aux hommes, accèdent peu aux postes de responsabilité (Loua, 2018). Lorsque le village n'a pas d'école, il faut envoyer les enfants dans un autre. Cela entraîne des frais non soutenables pour beaucoup de familles qui, contraintes de choisir, donnent souvent la priorité à leurs fils, considérés comme devant prendre, un jour, la relève du chef de famille. De plus, le temps consacré à se rendre à l'école ne permet plus aux filles de s'acquitter des tâches ménagères qui leur sont dévolues. Bien que les droits des femmes soient institutionnellement reconnus au Mali, les nombreuses incitations à assurer leur inclusion et leur réussite scolaires ne sont pas toujours suivies d'effet. Malgré la création de différents dispositifs (comme la Cellule nationale pour la promotion de la scolarisation des filles en 1991 ou la Politique nationale genre de 2013), il est possible, sans opposition frontale, de ne pas appliquer les réformes en continuant à s'appuyer sur des pratiques que l'interférence de normes, venues tout autant de l'époque coloniale que de structures traditionnelles ou de préconisations de l'Unesco et des enquêtes internationales, permet de pérenniser (Loua, id.). Si, en France, les jeunes filles accèdent, depuis 1924, au même baccalauréat que les garçons, elles constituent néanmoins toujours, dans l'entre-deux-guerres, un groupe limité aux zones urbaines et leurs professeurs sont essentiellement des femmes, dotées de diplômes différents de ceux de leurs collègues masculins (Héry, 2006). Malgré les succès incontestables de la scolarisation des filles dans les disciplines où elles étaient minoritaires (comme le droit, la médecine ou la gestion), leur présence s'accroît dans les filières traditionnellement les plus féminisées (lettres et sciences humaines) mais progresse très peu, à l'exception du domaine de la santé, dans les filières scientifiques et techniques. Malgré leur meilleure réussite éducative, elles continuent de s'orienter vers les filières les moins prestigieuses et rentables sur le marché du travail (Epiphane, 2017). Entre 1984 et 2013, cinq conventions interministérielles visent pourtant à améliorer l'orientation des garçons et des filles, notamment par l'augmentation de l'accès de celles-ci aux filières techniques et scientifiques porteuses d'emplois. L'éducation nationale pour sa part, avec des campagnes comme « Les métiers n'ont pas de sexe » ou "C'est technique, c'est pour elle ", ne cesse, dans la même période, de développer l'idée que la diversification de l'orientation des jeunes filles est productrice d'égalité (Epiphane, 2019).

\section{DES IMAGINAIRES ÉDUCATIFS}

Dans les deux cas de succès relatifs évoqués, l'enjeu ne semble pas tant la cohérence des réformes que leur acceptabilité. Les obstacles matériels sont évidemment nombreux à l'échelle de chaque société, mais les imaginaires éducatifs des acteurs semblent concourir très fortement au destin des mesures adoptées.

Dans le cas du Mali, la volonté d'utiliser tous les talents pour moderniser le pays semble surtout contrecarrée par les décalages qui perdurent entre les intentions des élites influencées par des normes extra-nationales et les manières traditionnelles de concevoir et mettre en ouvre l'éducation. Dans le cadre d'une certaine conception de l'islam, principale religion du pays, la femme doit se marier tôt, avoir de nombreux enfants, se cantonner à la sphère privée. À quoi bon, alors, donner aux jeunes filles une formation dont ni elles ni la société ne tireront parti ? 
Les grossesses précoces, souvent non désirées, suscitent, dans les écoles rurales, de nombreux décrochages scolaires. Les enseignants eux-mêmes peuvent être porteurs de stéréotypes de genre qui contrecarrent leur propre travail. Les manuels en vigueur peuvent contribuer à l'intériorisation de son infériorité lorsque, comme le livre de lecture Mamadou et Binéta, édité en France et utilisé par l'école malienne, ils mettent en scène des personnages masculins toujours plus nombreux et porteurs de meilleurs rôles que les personnages féminins (Loua, 2019). De fait, travailler à opérationnaliser des réformes semble passer aujourd'hui par l'appui sur des acteurs intermédiaires comme les ONG Agir et Save the children, qui ont impliqué dans leur programme les grand-mères (qui sont, au Mali, les confidentes des jeunes filles), les agents de santé, les associations de jeunesses villageoises et les enseignants (Loua, 2019).

Dans le cas de la France, l'adoption de mesures en faveur de la scolarisation des filles n'est véritablement devenue effective que lorsque la société dans son ensemble, notamment du fait de la contribution des femmes à l'effort de guerre, a mis en évidence leurs compétences et suscité l'ambition scolaire à leur égard. À l'inverse, leur progression dans les parcours d'études rencontre le fameux «plafond de verre » que constitue la division genrée du travail et des responsabilités sociales et politiques. Pour accéder au baccalauréat, les jeunes filles françaises ont dû se frayer un chemin à travers de persistants stéréotypes de genre. De constitution plus faible, les adolescentes étaient-elles vraiment adaptées à un travail intellectuel qui allait dessécher et épuiser les mères de demain (Héry, 2006) ? À l'heure décisive des choix d'orientation, il convient de prouver à soi-même et aux autres qu'on est bien une fille " féminine » ou un garçon " masculin » (Vouillot, 2014). Une fois le diplôme en poche, les jeunes femmes connaissent aujourd'hui encore le " gaspillage social» que représente l'écart entre performances scolaires et insertion socioprofessionnelle (Duru-Bellat, 1990).

Le sort des réformes éducatives se joue en partie dans le décalage qui existe entre les idées des législateurs et l'imaginaire de l'ensemble des acteurs. La sociologie du laboratoire nous apprend que les découvertes scientifiques les plus conceptuellement robustes peuvent dépérir dans des dossiers, si elles ne sont mises en œuvre par des personnes qui en voient et en partagent l'intérêt (Akrich et al., 2006). Il en va sans doute de même des réformes éducatives qui peuvent très bien ne s'appliquer que partiellement, voire pas du tout, si ceux qui sont censés les faire vivre ont des raisons de penser qu'elles leur sont étrangères. Si c'est le cas, leurs propres réticences à changer leurs pratiques se nourrissent du soupçon de l'illégitimité de ce qui leur est prescrit et la tentation de rajouter des réformes aux réformes pour accélérer le cours des choses peut alors conforter les statu quo.

$\mathrm{Si}$, dans beaucoup de pays, la scolarisation des filles va croissant, beaucoup d'inégalités les frappent néanmoins, tant dans la durée, la qualité que la rentabilité de leurs études. C'est qu'il n'est pas possible de changer l'école sans toucher aussi à l'ensemble des rapports sociaux. Lorsque les enseignants de l'école dite maternelle sont massivement des femmes et les professeurs d'université des hommes, il est diffi- 
cile de faire admettre aux élèves que la scolarisation est la clé de l'égalité sociale. Des démarches intersectionnelles, articulant les questions du genre et celles des rapports sociaux de domination, sont de nature à éclairer en la matière une action réformatrice qui, lorsqu'elle confond le prescrit et le réel, risque de semer la désillusion et de s'épuiser elle-même.

\section{BiBLIOGRAPHIE}

AKRICH M., CALLON M. et LATOUR B. (éd.) (2006). Sociologie de la traduction : textes fondateurs. Paris : Mines ParisTech, les Presses, « Sciences sociales».

DURU-BELLAT M. (1990). L'école des filles. Quelles formations pour quels rôles sociaux? Paris : L'Harmattan.

EPIPHANE D. (2019). "Les politiques publiques en faveur des scolarités féminines en France : entre mouvements de fond et résistances sociales». Colloque "Conditions de réussite des réformes en éducation ", 12-14 juin, CIEP. [En ligne]. Revue internationale d'éducation de Sèvres. [https://journals.openedition.org/ries/7274]

EPIPHANE D. (2017). "Genre et éducation », dans A. Van Zanten et P. Rayou (dir.), Dictionnaire de l'éducation, $2^{\mathrm{e}}$ édition, Presses Universitaires de France, p. 449-454.

HERY É (2003). «Quand le baccalauréat devient mixte », Clio. Histoire, femmes et sociétés. [En ligne], $\mathrm{n}^{\circ}$ 18. [http://journals.openedition.org/clio/612] ; DOI : 10.4000/clio.612

LOUA S. (2019). "La question de la réussite scolaire des filles au Mali ». Colloque «Conditions de réussite des réformes en éducation », 12-14 juin, CIEP. [En ligne]. Revue internationale d'éducation de Sèvres. [https://journals.openedition.org/ries/7310]

LOUA S. (2018). "État des lieux de l'éducation des filles et des femmes au Mali : contraintes et défis ", Revue internationale d'éducation de Sèvres, $\mathrm{n}^{\circ}$ 78, p. 103-113. [DOI : https://doi.org/10.4000/ries.6571]

VOUILLOT F. (2014). Les métiers sont-ils un sexe? Pour sortir des sentiers battus de l'orientation des filles et des garçons. Paris : éditions Belin. 